\title{
Fluctuaciones bursátiles de las acciones de empresas mineras peruanas durante el Estado de Emergencia Nacional por efecto de la COVID-19
}

\section{Stock market fluctuations of the actions of peruvian mining companies during the state of national emergency due to the effect of COVID-19}

\author{
Andrea Carmen Lidia Ordinola Valdez \\ Consorcio de Minería y Construcción VULCANO S.A.C. \\ Tacna, Perú \\ andreaordinolav@gmail.com \\ https://orcid.org/0000-0003-1802-8522 \\ José Alejandro Filinich Cutipa \\ Colegio de Economistas de Moquegua \\ Moquegua, Perú \\ jafilinich@gmail.com \\ https://orcid.org/0000-0001-6421-8381 \\ [Presentado: 15/05/2021, Aceptado: 25/06/2021]
}

\section{RESUMEN}

El objetivo del trabajo fue describir las repercusiones que se han venido suscitando a partir del Estado de Emergencia Nacional por efectos del coronavirus en las acciones de las empresas mineras peruanas. La investigación fue de enfoque cuantitativo, de nivel explicativo (causal), basada en la cuantificación y el análisis econométrico de las series de tiempo de activos financieros que cotizan en la Bolsa de Valores de Lima (BVL), durante el periodo de cuarentena, iniciada el 16 de marzo y concluida el 30 de junio del 2020, y su comportamiento poscuarentena. La muestra en estudio estuvo conformada por las compañías mineras representativas que se encuentran en el top ten de las más importantes a nivel nacional. Se concluye que la rentabilidad de las mencionadas empresas que cotizan en la BVL, en el periodo estudiado, ha sido negativa.

Palabras clave: Mercado de valores, minería, rentabilidad.

\begin{abstract}
The objective of the work was to describe the repercussions that have been arising from the State of Nacional Emergency due to the effects of the coronavirus on the actions of Peruvian mining companies. The research was a quantitative approach, of an explanatory (causal) level, based on the quantification and econometric analysis of the time series of financial assets listed on the Lima Stock Exchange, focusing on the quarantine period that began on March 16 and ended on June 30, 2020, and its postquarantine behavior. The selected sample was made up of representative mining companies that are in the top ten of the most important nationally. It is concluded that the profitability of mining companies listed on the Lima Stock Exchange, in the period studied, has been negative.
\end{abstract}

Keywords: Stock market, mining, profitability. 


\section{INTRODUCCIÓN}

La seria crisis sanitaria producto de la pandemia ocasionada en Wuhan, China central, está afectando la economía global. Y uno de los sectores más afectados es la minería, no solo por la recesión existente y la caída de la demanda de los metales a nivel mundial, sino por la dificultad de establecer los protocolos sanitarios exigidos para retomar actividades, principalmente para poner en práctica el distanciamiento social, esos son los retos que actualmente enfrenta el sector minero en el país. La Sociedad Nacional de Minería, Petróleo y Energía calculó que la mayor parte de las compañías dedicadas al sector en el Perú se encontraban suspendidas temporalmente (Saldarriaga, 2020). Ya que es un sector en la que la alternativa del home office no será sostenible en el tiempo como para repotenciar sus actividades, se tendría que adecuar ciertas medidas de prevención que proteja al capital humano y que, a su vez no afecte en gran medida la producción. Puesto que China es nuestro principal cliente con cerca del $45 \%$ de nuestras exportaciones mineras, se estimó que el país tendría una reducción en el crecimiento del PBI en el 2020 en comparación con el año anterior (Redacción EC, 2020). Además, las exportaciones de cobre sufrieron una caída de $26.3 \%$ en relación con el periodo similar del año anterior (Redacción Gestión, 2020).

A nuestro país le ha tocado enfrentar esta situación con una economía de lento crecimiento, dado que el año 2019 la tasa de crecimiento fue $2.2 \%$, y de acuerdo a la 'Regla del 70', duplicar nuestro ingreso tomaría 32 años. Estos grandes efectos a largo plazo son producto de las diferencias de las tasas de crecimiento anual, las cuales son ocasionadas por las tasas de crecimiento compuestas (Larraín \& Sachs, 2002). El Fondo Monetario Internacional (FMI) estimó que, en nuestro país, el PBI caería en $4.5 \%$, como consecuencia de la emergencia sanitaria (Prialé, 2020). El Ministerio de Energía y Minas manifestó que las inversiones mineras registraron una contracción de $25 \%$ en el primer semestre del año, a comparación del periodo similar del año anterior (Redacción EC, 2020).

En base al contexto actual que se ha descrito y a la información bursátil de las compañías mineras que cotizan en la BVL, se ha procedido a la elección de las tres empresas mineras más representativas del país, de las cuales se ha procedido a extraer la data desde el primer día hábil del año 2017 hasta agosto del 2020, con la finalidad de ver con claridad las fluctuaciones acontecidas desde antes de los sucesos ocasionados por la pandemia, enfocándonos en el periodo del Estado de Emergencia por efectos de la COVID-19, y poscuarentena.

\section{MATERIAL Y MÉTODOS}

La metodología econométrica es importante para el análisis de la evolución de los índices bursátiles durante el periodo del Estado de Emergencia Nacional, iniciado el 16 de marzo del 2020 y que finalizó el 30 de junio del mismo año. Para ello se consideró el periodo poscuarentena, ya que en algunos departamentos y provincias la cuarentena se retomó de manera focalizada.

En primer lugar, se ha establecido los índices bursátiles de las principales compañías mineras que han sido seleccionadas por mantener sus cotizaciones en la BVL, con el objeto de realizar un análisis de la situación actual y de las fluctuaciones en el periodo ya mencionado, y de la misma manera, poder establecer la relación rentabilidad-riesgo.

En segundo lugar, se ha procedido a evaluar los supuestos de no autocorrelación, mediante el modelo de regresión lineal y la prueba de Breusch-Godfrey, hasta con tres rezagos, con la finalidad de demostrar su significancia y su correlación hasta de tercer orden. También, se ha procedido a evaluar el supuesto de no multicolinealidad, con el factor de inflación de las varianzas, y la homocedasticidad con la prueba White con términos cruzados y la prueba $\mathrm{ARCH}$.

Esta investigación pretende analizar la proyección ceteris paribus, además de la situación actual y la trayectoria de los índices bursátiles de las compañías mineras, planteando una comparación entre el riesgo y la rentabilidad. 


\section{RESULTADOS}

Entre las principales empresas mineras en Perú podemos mencionar: Antamina, Cerro Verde, Las Bambas, Southern Perú Cooper, Antapaccay, Buenaventura y Minsur. Y en el presente trabajo se ha analizado las fluctuaciones bursátiles de tres compañías mineras que cotizan en la BVL, las cuales figuran entre las diez más importantes del país.

En la Figura 1 se puede observar, un decrecimiento de las cotizaciones desde fines del mes de febrero del 2020, estos indicadores negativos se debieron por dos razones, la primera, por la caída del precio de los principales commodities y, la segunda, por el impacto de las medidas establecidas como consecuencia de la emergencia sanitaria. También se aprecia una gran diferenciación entre ellas, con una ligera tendencia negativa, a pesar de la escasa cotización de sus acciones en la BVL, y que con el transcurso del tiempo y de las medidas que el Gobierno adopte en beneficio de las mencionadas compañías mejore el panorama actual.

\section{Figura 1}

Cotizaciones de las compañías mineras en Perú

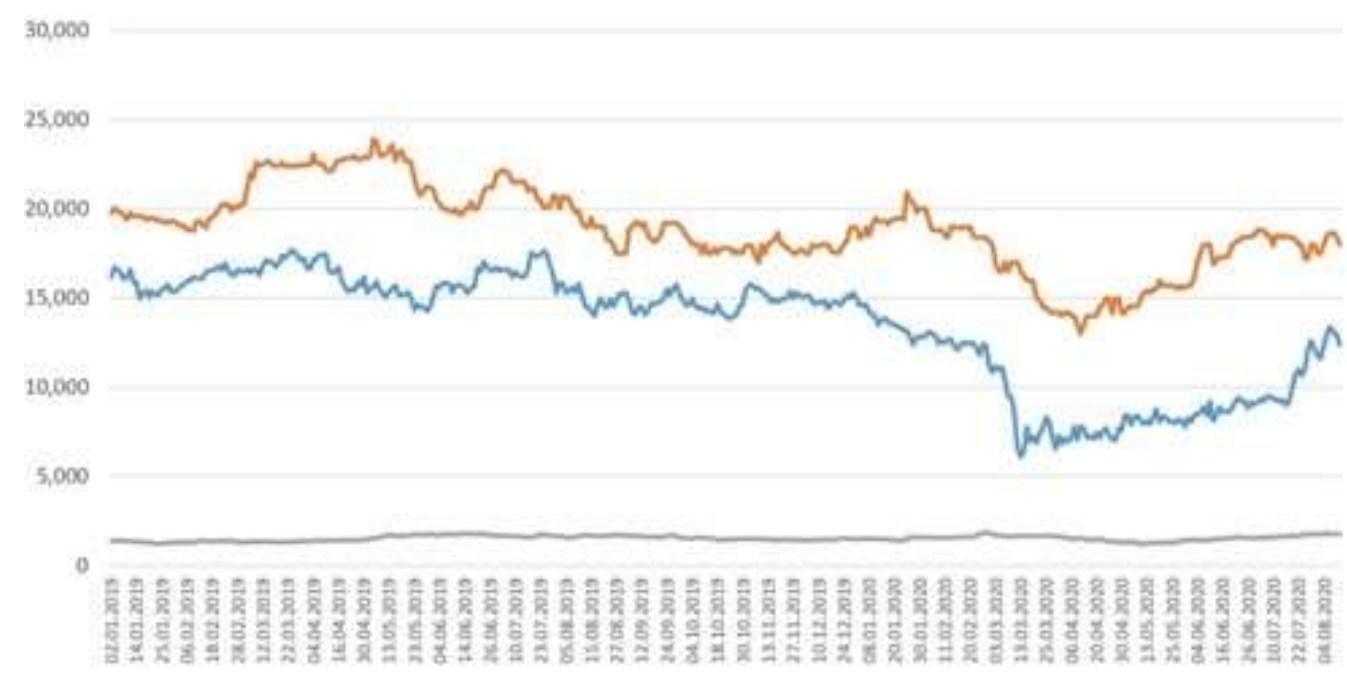

Fuente: Elaboración propia en base a la data de Investing.com

Figura 2

Frontera eficiente de las compañías mineras en Perú

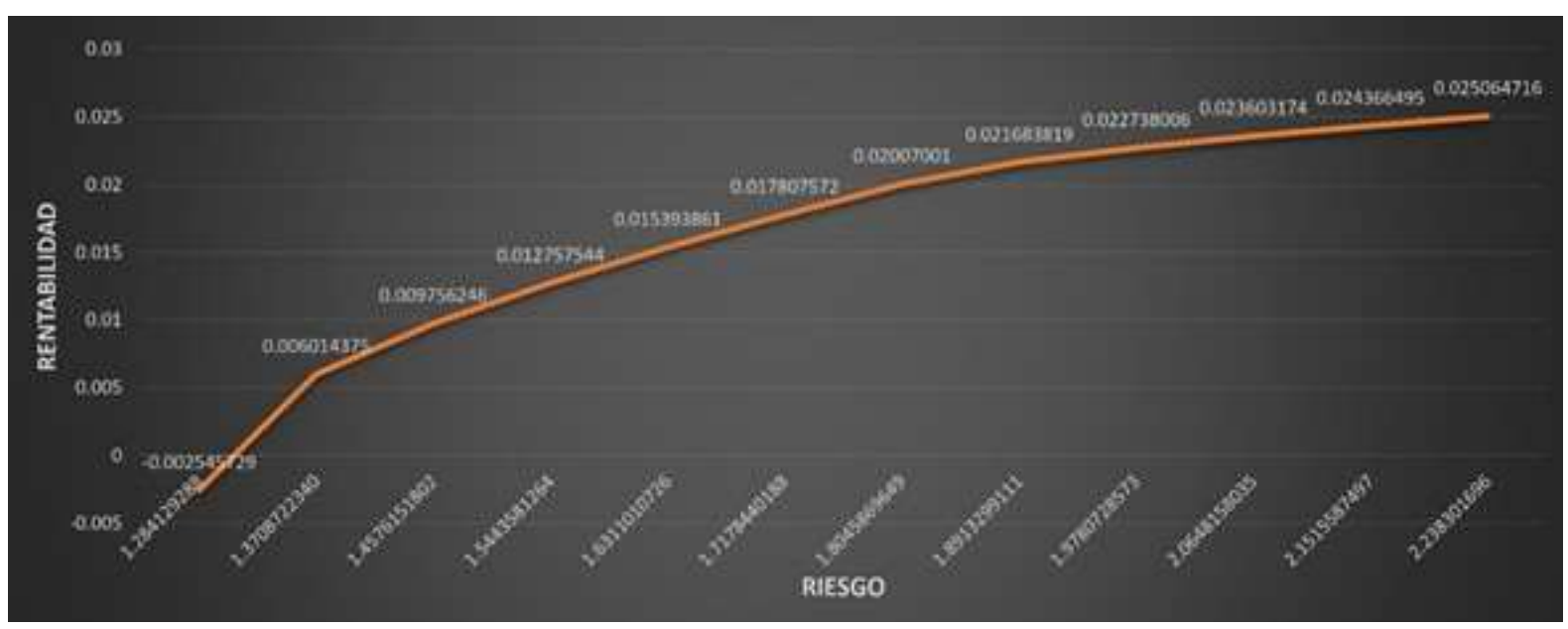

Fuente: Elaboración propia en base a la data de Investing.com 
$\mathrm{Al}$ tener en cuenta la data actual, (Figura 2) en la que la rentabilidad de las grandes compañías mineras se ha visto afectada, en referencia a los riesgos que se han venido suscitando, se observa que el panorama no es muy alentador, pero existe una leve tendencia a mejorar, de acuerdo a las proyecciones presentadas por el FMI para nuestro país, las cuales indican que, a pesar de haber tenido una desaceleración en la economía, para el 2021 se podría tener un crecimiento considerable. En la frontera eficiente relaciona la mejor rentabilidad para un determinado riesgo, mientras mayor sea el riesgo mayor será la rentabilidad.

A continuación, se realizó el modelo de regresión lineal en base a la data de las compañías que conforman la muestra, las cuales han sido afectadas por los efectos de la COVID-19.

\section{Tabla 1}

Modelo de regresión de las principales empresas mineras en la BVL

\section{Dependent variable: BVL}

Method: Least Squares

Sample: 1757

Included observations: 757

\begin{tabular}{ccccc}
\hline \multicolumn{1}{c}{ Variable } & Coefficient & Std. Error & t-Statistic & Prob. \\
\hline C & 6098.135 & 356.8508 & 17.08875 & 0.0000 \\
X & 0.634619 & 0.019144 & 33.15028 & 0.0000 \\
Y & -0.034612 & 0.013892 & -2.491531 & 0.0129 \\
Z & 3.128019 & 0.251157 & 12.45442 & 0.0000 \\
Adjusted R-squared & 0.714275 & S.D. dependent var & & 18831.57 \\
S.E. of regression & 1031.116 & Akaike info criterion & & 1929.004 \\
Sum squared resid & $8.01 \mathrm{E}+08$ & Schwarz criterion & & 16.71994 \\
Log likelihood & -6324.497 & Hannan-Quinn criter & & 16.74440 \\
F-statistic & 630.9660 & Durbin-Watson stat & & 16.72936 \\
Prob(F-statistic) & 0.000000 & & & \\
\hline
\end{tabular}

Fuente: Elaboración propia en base a la data de Investing.com

El estadístico Durbin-Watson es 0.046029 , por lo tanto, se encuentra en la región de autocorrelación positiva de primer orden. De la misma manera, se procede a realizar la evaluación mediante la prueba de Breusch-Godfrey. Estos resultados permiten demostrar la relación que existe de la data a lo largo del tiempo. 


\section{Tabla 2}

Correlación Serial Breusch-Godfrey con un rezago

\begin{tabular}{llll}
\hline F-statistic & 15371.93 & Prob. F(1,752) & 0.0000 \\
Obs*R-squared & 721.6945 & Prob. Chi-square(1) & 0.0000 \\
\hline
\end{tabular}

Test Equation:

Dependent variable: RESID

Method: Least Squares

Sample: 1757

Included observations: 757

Presample missing value lagged residuals set to zero

\begin{tabular}{ccccc}
\hline \multicolumn{1}{c}{ Variable } & Coefficient & Std. Error & t-Statistic & Prob. \\
C & 108.5111 & 77.12173 & 1.407011 & 0.1598 \\
X & -0.008590 & 0.004138 & -2.076018 & 0.0382 \\
Y & 0.002149 & 0.003002 & 0.715772 & 0.4744 \\
Z & -0.025639 & 0.054276 & -0.472387 & 0.6368 \\
RESID(-1) & 0.978216 & 0.007890 & 123.9836 & 0.0000 \\
\hline R-squared & 0.953361 & Mean dependent var & & $-6.70 \mathrm{E}-13$ \\
Adjusted & 0.953113 & S.D. dependent var & & 1029.068 \\
R-squared & 222.8279 & Akaike info criterion & & 13.65726 \\
S.E. of regression & 37338498 & Schwarz criterion & & 13.68784 \\
Sum squared resid & -5164.273 & Hannan-Quinn criter & & 13.66904 \\
Log likelihood & 3842.981 & Durbin-Watson stat & & 2.204542 \\
F-statistic & 0.000000 & & & \\
Prob(F-statistic) & & & \\
\hline
\end{tabular}

Fuente: Elaboración propia en base a la data de Investing.com

La probabilidad resultante es menor a 0.05 , por lo tanto, existe correlación de primer orden, y los errores rezagados de un periodo son significativos, confirmando el resultado del estadístico de Durbin-Watson. 


\section{Tabla 3}

Correlación Serial Breusch-Godfrey con dos rezagos

\begin{tabular}{llll}
\hline F-statistic & 7816.154 & Prob. F(2,751) & 0.0000 \\
Obs*R-squared & 722.2996 & Prob. Chi-square(2) & 0.0000 \\
\hline
\end{tabular}

Test Equation:

Dependent variable: RESID

Method: Least Squares

Sample: 1757

Included observations: 757

Presample missing value lagged residuals set to zero

\begin{tabular}{ccccc}
\hline Variable & Coefficient & Std. Error & t-Statistic & Prob. \\
\hline C & 121.7502 & 76.59620 & 1.589507 & 0.1124 \\
Y & -0.009620 & 0.004115 & -2.337926 & 0.0197 \\
Z & 0.002422 & 0.002979 & 0.813089 & 0.4164 \\
RESID(-1) & -0.029128 & 0.053854 & -0.540872 & 0.5888 \\
RESID(-2) & 0.850497 & 0.036149 & 23.52759 & 0.0000 \\
\hline R-squared & 0.131145 & 0.036238 & 3.619013 & 0.0003 \\
Adjusted & 0.954161 & Mean dependent var & & $-6.70 \mathrm{E}-13$ \\
R-squared & 0.953855 & S.D. dependent var & & 1029.068 \\
S.E. of regression & 221.0569 & Akaike info criterion & & 13.64261 \\
Sum squared resid & 36698486 & Schwarz criterion & & 13.67930 \\
Log likelihood & -5157.729 & Hannan-Quinn criter & & 13.65674 \\
F-statistic & 3126.461 & Durbin-Watson stat & & 1.963352 \\
Prob(F-statistic) & 0.000000 & & & \\
\hline
\end{tabular}

Fuente: Elaboración propia en base a la data de Investing.com

Con dos rezagos se observa también que hay autocorrelación de primer y segundo orden, por lo que ambos son significativos. 
Tabla 4

Correlación Serial Breusch-Godfrey con tres rezagos

\begin{tabular}{llll}
\hline F-statistic & 5229.410 & Prob. F(3,750) & 0.0000 \\
Obs*R-squared & 722.4616 & Prob. Chi-square(3) & 0.0000 \\
\hline
\end{tabular}

Test Equation:

Dependent variable: RESID

Method: Least Squares

Sample: 1757

Included observations: 757

Presample missing value lagged residuals set to zero

\begin{tabular}{|c|c|c|c|c|}
\hline Variable & Coefficient & Std. Error & t-Statistic & Prob. \\
\hline $\mathrm{C}$ & 128.7603 & 76.55942 & 1.681835 & 0.0930 \\
\hline$X$ & -0.010085 & 0.004115 & -2.450791 & 0.0145 \\
\hline $\mathrm{Y}$ & 0.002527 & 0.002975 & 0.849403 & 0.3959 \\
\hline $\mathrm{Z}$ & -0.031141 & 0.053774 & -0.579110 & 0.5627 \\
\hline $\operatorname{RESID}(-1)$ & 0.841665 & 0.036394 & 23.12625 & 0.0000 \\
\hline $\operatorname{RESID}(-2)$ & 0.073219 & 0.047567 & 1.539286 & 0.1242 \\
\hline $\operatorname{RESID}(-3)$ & 0.068400 & 0.036469 & 1.875534 & 0.0611 \\
\hline R-squared & 0.954375 & Mean dependent var & & $-6.70 \mathrm{E}-13$ \\
\hline $\begin{array}{l}\text { Adjusted } \\
\text { R-squared }\end{array}$ & 0.954010 & S.D. dependent var & & 1029.068 \\
\hline S.E. of regression & 220.6873 & Akaike info criterion & & 13.64057 \\
\hline Sum squared resid & 36527167 & Schwarz criterion & & 13.68338 \\
\hline Log likelihood & -5155.958 & Hannan-Quinn criter & & 13.65706 \\
\hline F-statistic & 2614.705 & Durbin-Watson stat & & 1.948820 \\
\hline Prob(F-statistic) & 0.000000 & & & \\
\hline
\end{tabular}

Fuente: Elaboración propia en base a la data de Investing.com

Se afirma, además, que el modelo cumple con el supuesto de no multicolinealidad exacta, facilitando aislar los efectos individuales de cada uno de los estimadores sobre la variable dependiente. También se evalúa con el factor de inflación de las varianzas. 
Tabla 5

Factor de inflación de las varianzas

Variance inflation factors

Sample: 1757

Included observations: 757

\begin{tabular}{cccc}
\hline Variable & $\begin{array}{c}\text { Coefficient } \\
\text { variance }\end{array}$ & $\begin{array}{c}\text { Uncentered } \\
\text { VIF }\end{array}$ & $\begin{array}{c}\text { Centered } \\
\text { VIF }\end{array}$ \\
\hline $\mathrm{C}$ & 127342.5 & 90.66808 & NA \\
$\mathrm{X}$ & 0.000366 & 50.16189 & 1.314144 \\
$\mathrm{Y}$ & 0.000193 & 65.03403 & 1.536384 \\
$\mathrm{Z}$ & 0.063080 & 106.8764 & 1.359393 \\
\hline
\end{tabular}

Fuente: Elaboración propia en base a la data de Investing.com

Tabla 6

Prueba de heterocedasticidad: White, con términos cruzados

\begin{tabular}{llll}
\hline F-statistic & 32.27781 & Prob. F(9,747) & 0.0000 \\
Obs*R-squared & 211.9602 & Prob. Chi-square(9) & 0.0000 \\
Scaled explained SS & 208.2031 & Prob. Chi-square(9) & 0.0000 \\
\hline
\end{tabular}

Test Equation:

Dependent variable: $\operatorname{RESID}^{\wedge} 2$

Method: Least Squares

Sample: 1757

Included observations: 757

Presample missing value lagged residuals set to zero

\begin{tabular}{ccccc}
\hline Variable & Coefficient & Std. Error & t-Statistic & Prob. \\
$\mathrm{C}$ & -18299847 & 4221075. & -4.335352 & 0.0000 \\
$\mathrm{X}^{\wedge} 2$ & -0.079829 & 0.013967 & -5.715453 & 0.0000 \\
$\mathrm{X}^{*} \mathrm{Y}$ & 0.038579 & 0.015974 & 2.415119 & 0.0160 \\
$\mathrm{X}^{* \mathrm{Z}}$ & 0.251178 & 0.201623 & 1.245784 & 0.2132 \\
$\mathrm{X}$ & 657.1830 & 258.0868 & 2.546364 & 0.0111 \\
$\mathrm{Y}^{\wedge} 2$ & -0.036629 & 0.008535 & -4.291324 & 0.0000 \\
$\mathrm{Y}^{* \mathrm{Z}}$ & 0.086246 & 0.162435 & 0.530958 & 0.5956 \\
$\mathrm{Y}$ & 1009.495 & 230.5619 & 4.378413 & 0.0000 \\
$\mathrm{Z}^{\wedge} 2$ & -4.625252 & 2.045635 & -2.261035 & 0.0240 \\
$\mathrm{Z}$ & 8286.634 & 5123.989 & 1.617223 & 0.1063 \\
\hline R-squared & 0.280000 & Mean dependent var & & 1057582 \\
Adjusted R-squared & 0.271326 & S.D. dependent var & & 1491191 \\
S.E. of regression & 1272917 & Akaike info criterion & & 30.96464 \\
Sum squared resid & $1.21 \mathrm{E}+15$ & Schwarz criterion & & 31.02580 \\
Log likelihood & -11710.12 & Hannan-Quinn criter & & 30.98820 \\
F-statistic & 32.27781 & Durbin-Watson stat & & 0.183126 \\
Prob(F-statistic) & 0.000000 & & \\
\hline
\end{tabular}

Fuente: Elaboración propia en base a la data de Investing.com 
Las probabilidades son menores a 0.05 , por lo tanto, el modelo de homocedasticidad no se cumple para una prueba White con términos cruzados.

Tabla 7

Prueba ARCH (Autoregressive conditional heteroskedaticity)

\begin{tabular}{llll}
\hline F-statistic & 6407.708 & Prob. F(1,754) & 0.0000 \\
Obs*R-squared & 676.4067 & Prob. Chi-square(1) & 0.0000 \\
\hline
\end{tabular}

Test Equation:

Dependent variable: $\operatorname{RESID}^{\wedge} 2$

Method: Least Squares

Sample: 2757

Included observations: 756 after adjustments

\begin{tabular}{lcccc}
\hline \multicolumn{1}{c}{ Variable } & Coefficient & Std. Error & t-Statistic & Prob. \\
\hline \multicolumn{1}{c}{ C } & 58363.69 & 21593.39 & 2.702850 & 0.0070 \\
\multicolumn{1}{c}{ RESID^2(-1) } & 0.946649 & 0.011826 & 80.04816 & 0.0000 \\
\hline R-squared & 0.894718 & Mean dependent var & 1057473 \\
Adjusted R-squared & 0.894578 & S.D. dependent var & 1492175 \\
S.E. of regression & 484490.2 & Akaike info criterion & 29.02222 \\
Sum squared resid & $1.77 \mathrm{E}+14$ & Schwarz criterion & 29.03447 \\
Log likelihood & -10968.40 & Hannan-Quinn criter & 29.02694 \\
F-statistic & 6407.708 & Durbin-Watson stat & 2.345184 \\
Prob(F-statistic) & 0.000000 & & \\
\hline
\end{tabular}

Fuente: Elaboración propia en base a la data de Investing.com

La probabilidad es menor a 0.05 , con lo cual tenemos un modelo de heterocedasticidad tipo $\mathrm{ARCH}$, la varianza del periodo anterior tiene influencia en la varianza actual.

\section{DISCUSIÓN}

El objetivo de la investigación es analizar la evolución de los índices bursátiles de las principales compañías mineras existentes en el país y que, a su vez cotizan sus acciones en la BVL, en el periodo correspondiente al Estado de Emergencia Nacional por efectos de la COVID-19, iniciado el 16 de marzo del 2020 y finalizado el 30 de junio del mismo año. Se consideró también la poscuarentena, a pesar que algunas provincias y distritos retomaron la cuarentena de manera focalizada. 
El estudio se origina a partir de las repercusiones que afronta la economía nacional por las medidas restrictivas establecidas por el gobierno por efectos de la COVID-19, ya que la minería es uno de los sectores más afectados, se observa una caída significativa de dos de las tres empresas estudiadas, además, podemos afirmar que dicha caída inicia desde el mes de febrero, un mes antes del inicio de la cuarentena nacional.

El modelo no cumple con el supuesto de autocorrelación, en vista de que el estadístico DurbinWatson da como resultado 0.046029 , por lo tanto, los errores del modelo se relacionan, afirmación que se evidencia con la prueba de Breusch-Godfrey. Además, el modelo cumple con el supuesto de no multicolinealidad exacta, ya que una vez obtenido el modelo de regresión lineal se ha procedido a evaluar con el factor de inflación de las varianzas, donde todos los resultados son menores a 10, lo que significa que no hay problema de multicolinealidad. Finalmente, podemos afirmar que se trata de un modelo heterocedástico, comprobándose con las pruebas White y $\mathrm{ARCH}$, es decir, las acciones de las empresas mineras durante el periodo estudiado son fluctuantes.

El presente estudio concluye que se debe priorizar las actividades relacionadas al sector y repotenciar la minería, a pesar de las fluctuaciones bursátiles que se han venido suscitando en el periodo de cuarentena y que han afectado a las compañías, para ello se debe tener en cuenta que el precio global de los commodities estaría por debajo del precio que se ha mantenido hasta antes de la propagación del virus. El gobierno busca crear un fondo con la finalidad que estabilice los recursos del canon minero y protegerlos frente a las fluctuaciones de los precios de los metales a nivel internacional (Redacción Gestión, 2020). Se recomienda ejecutar planes de contingencia para evitar consecuencias similares futuras que afecten al sector, en base a los resultados obtenidos. Y de la misma manera, las empresas tendrán que desarrollar la implementación de protocolos sanitarios que brinden protección al factor humano, y priorizar el enfoque estratégico de la gestión del talento humano, ya que es una parte importante de la gerencia de los recursos humanos en todas las organizaciones.

\section{REFERENCIAS}

Investing.com. (s.f.). Investing.com.

Larraín, F. \& Sachs, J. (2002). Macroeconomía en la economía global (Segunda edición ed.). Buenos Aires: PRENTICE HALL Y PEARSON EDUCATION S.A.

Prialé, J. (14 de 04 de 2020). FMI estima que economía peruana caería $4.5 \%$ en 2020 pero rebotaría a crecer 5.2\% en 2021. Diario Gestión. https://gestion.pe/economia/fmi-estima-que-economia-peruanacaeria-45-en-2020-pero-rebotaria-a-crecer-52-en-2021-noticia/\#: :text=Econom\%C3\%ADa,FMI\% 20estima\% 20que\% 20econom\% C3\% ADa\% 20peruana\%20caer\%C3\% ADa\% 20 $4.5 \% 25 \% 20$ en $\% 202020 \% 20$ pero,a $\% 20$ crecer\%205.2\%25\%20

Redacción EC. (21 de 04 de 2020). Coronavirus en Perú: Precios bajos de cobre y oro desalientan inversiones exploratorias. Diario El Comercio. https://elcomercio.pe/economia/peru/coronavirusen-peru-precios-bajos-de-cobre-y-oro-desalientan-inversiones-exploratorias-pwc-mineriacommodities-nndc-noticia/.

Redacción EC. (04 de 08 de 2020). Inversiones mineras caen 25\% en primer semestre al sumar US\$ 1.910 millones. Diario El Comercio. https://elcomercio.pe/economia/peru/inversiones-mineras- 
caen-25-en-primer-semestre-del-ano-al-sumar-us-1910-millones-minem-nndc-noticia/.

Redacción Gestión. (12 de 08 de 2020). Exportaciones de cobre registran caída de $26.3 \%$ en primer semestre del 2020, señala la SNMPE. Diario Gestión. https://gestion.pe/economia/exportacionesde-cobre-registran-caida-de-263-en-primer-semestre-del-2020-senala-la-snmpe-nndc-noticia/.

Redacción Gestión. (11 de 08 de 2020). PCM: Plantea creación de fondo de estabilización del canon minero para estabilizar los recursos. Diario Gestión. https://gestion.pe/economia/voto-deconfianza-congreso-walter-martos-pcm-plantea-creacion-de-fondo-de-estabilizacion-del-canonminero-para-estabilizar-los-recursos-voto-de-confianza-nndc-noticia/.

Saldarriaga, J. (07 de 04 de 2020). Tecnología Minera. Diario El Comercio. https://elcomercio. pe/economia/dia-1/mineria-covid-19-su-impacto-en-la-mineria-luego-de-tres-semanas-decuarentena-covid-19-noticia/. 